\title{
Masking within and across visual dimensions: Psychophysical evidence for perceptual segregation of color and motion
}

\author{
SAMUEL W. CHEADLE AND SEMIR ZEKI \\ Wellcome Laboratory of Neurobiology, Anatomy Department, University College London, London, UK
}

(Received February 2, 2011; Accepted June 9, 2011; First Published Online August 11, 2011)

\begin{abstract}
Visual masking can result from the interference of perceptual signals. According to the principle of functional specialization, interference should be greatest when signal and mask belong to the same visual attribute (e.g., color or motion) and least when they belong to different ones. We provide evidence to support this view and show that the time course of masking is visual attribute specific. First, we show that a color target is masked most effectively by color (homogeneous target-mask pair) and least effectively by motion (heterogeneous pair) and vice versa for a motion target. Second, we show that the time at which the mask is most effective depends strongly on the target-mask pairing. Heterogeneous masking is strongest when the mask is presented before the target (forward masking) but this is not true of homogeneous masking. This finding supports a delayed cross-feature interaction due to segregated processing sites. Third, lengthening the stimulus onset asynchrony between target and mask leads to a faster improvement in color than in motion detectability, lending support for a faster color processing system and consistent with reports of perceptual asynchrony in vision. In summary, we present three lines of psychophysical evidence, all of which support a segregated neural coding scheme for color and motion in the human brain.
\end{abstract}

Keywords: Functional specialization, Visual masking, Psychophysics

\section{Introduction}

Our seemingly effortless ability to perceive a world in which all the different visual attributes are in apparently precise temporal and spatial registration belies a complex cortical machinery, which decomposes the visual image into constituents such as form, color and motion, and processes them in separate and specialized visual areas. The evidence for this functional specialization in the primate visual brain comes from anatomical, electrophysiological (Zeki, 1978; DeYoe \& van Essen, 1988; Livingstone \& Hubel, 1988; Zeki \& Shipp, 1988), and human imaging and clinical studies (Meadows, 1974; Zeki, 1990, 1991; Zeki et al., 1991; Zihl et al., 1991). This functional specialization has, moreover, temporal consequences since we perceive different attributes at different times, color taking temporal precedence over orientation, and orientation over motion (Moutoussis \& Zeki, 1997a,b; Zeki \& Moutoussis, 1997; Barbur et al., 1998; Arnold et al., 2001).

Of all the visual attributes, perhaps the easiest to separate both physiologically and perceptually are color and motion, color being associated with activity of the V4 complex and motion with activity of a separate system, based primarily on the area V5 (Zeki, 1978; Livingstone \& Hubel, 1988; Zeki et al., 1991). The evidence

Address correspondence and reprint requests to: Samuel W. Cheadle, Wellcome Laboratory of Neurobiology, Anatomy Department, University College London, London WC1E 6BT, UK. E-mail: s.cheadle@ucl.ac.uk in favor of the separation of motion and color also comes from psychophysical experiments, which show that motion detection is impaired under conditions of equiluminance (Ramachandran \& Gregory, 1978; Cavanagh et al., 1984), indicating that the motion system, although sensitive to chromatic signals, does not contain neurons tuned to specific hues (Gouras \& Kruger, 1979; Dobkins \& Albright, 1994). Additional psychophysical evidence is consistent with functional specialization for other visual dimensions (Krumhansl, 1984; Livingston \& Hubel, 1987; Theeuwes, 1992; Hong \& Shevell, 2006; Hong \& Blake, 2009).

In the study reported here, we investigate functional specialization psychophysically using a visual masking paradigm, by examining the strength of interference between two perceptual signals, either arising from the same visual attribute (homogeneous target-mask pairs) or from different ones (heterogeneous target-mask pairs). Masking refers to the impaired detectability of a target stimulus when immediately preceded or succeeded by a taskirrelevant visual input, referred to as the mask (Breitmeyer \& Ogmen, 2006). Visual temporal masking has been reported in both the motion (Braddick, 1973; Ferrera \& Wilson, 1987) and the color domain (Schmidt, 2002; Breitmeyer et al., 2004) but not across the two. Moreover, although masking of a target color with a color mask has been reported in two studies (Schmidt, 2002; Breitmeyer et al., 2004), both employed a metacontrast masking technique, in which the target and mask regions were nonspatially overlapping. Because this 
type of masking has been hypothesized to rely on a form of "motion deblurring" (Ansorge et al., 2007) rather than direct interference between target and mask signals, we chose to use the simplified backwards masking technique, in which the target and the mask overlap in space. This alone would enable us to draw conclusions regarding a functional specialization.

In our study, we manipulated the relationship between the target and mask, such that the target-mask pairing was either homogeneous (e.g., color target and color mask) or heterogeneous (e.g., color target and motion mask). If regions or cells in the visual system are nonspecialized and respond to multiple visual features (integrated representations), mask strength should remain constant across conditions (Fig. 1, Panel C). If cortical representations are exclusively integrated, it should be impossible to selectively mask one feature (e.g., color), while sparing the other (e.g., motion). This would not be true if the demonstrated functional specialization in the cortex is perceptually potent, that is, if signals from target and mask are processed in separate cortical sites or by different cells, when competition or interference will take place over a different time course, and is likely to be weaker (Fig. 1, Panel B).

Our study is divided into three experiments. In the first, we report the effect of homogeneous and heterogeneous target-mask pairs at both short and long stimulus onset asynchronies (SOAs); functional specialization predicts weaker masking in the case of heterogeneous pairs. In the second experiment, we investigate the time course of homogeneous pair masking in more detail, with the aim of exposing perceptual asynchronies between the visual features of color and motion. In the third section, we test the prediction that heterogeneous masking only occurs when the mask is given sufficient processing time (i.e., when the mask occurs prior to the target).

Our results constitute a psychophysical demonstration of functional specialization for the processing of color and motion in the human visual system.

\section{Experiment 1: Feature-selective masking}

Method

\section{Apparatus}

For all experiments, stimuli were displayed on a Sony Trinitron Multi-scan E450 monitor (refresh rate of $140 \mathrm{~Hz}$; Sony, Tokyo, Japan) and generated using the Cogent toolbox for MatLab on a windows XP machine.

\section{Stimuli and Procedure}

The target stimulus contained both color and motion, while the mask featured only a single attribute ${ }^{1}$. Stimuli were presented on a gray background $\left(6.9 \mathrm{~cd} / \mathrm{m}^{2}\right)$. The target was a fast moving (145 deg s ${ }^{-1}$; left or right) colored circle (Fig. 2). It was presented for $35 \mathrm{~ms}$ and covered a region of $5.1 \mathrm{deg}$. Two types of mask were tested, a color mask which consisted of a uniformly colored bar $(10.2 \times 5.1 \mathrm{deg}$; $200 \mathrm{~ms}$ duration; Fig. 3A) and a motion mask generated from the horizontal cyclic left-right motion of two fast moving white circles (Fig. 3B), covering the target region. The target colors were green and yellow, while the mask colors were red and blue ${ }^{2}$. Therefore, the target and mask colors could either be

\footnotetext{
${ }^{1}$ Salience was maximized for one visual feature dimension and minimized for the other. Motion was maximized by presenting a moving achromatic stimulus. Color was maximized by presenting a stationary chromatic stimulus.

${ }^{2}$ Color settings: green $(X=4.97, Y=9.95, Z=3.62)$, yellow $(X=7.24$, $Y=9.66, Z=3.4)$, red $(X=11.7, Y=6.79, Z=64.7)$, blue $(X=1.26, Y=$ $6.52, Z=64.7)$, and gray background $(X=6.75, Y=7.39, Z=13.3)$.
}

Target Mask

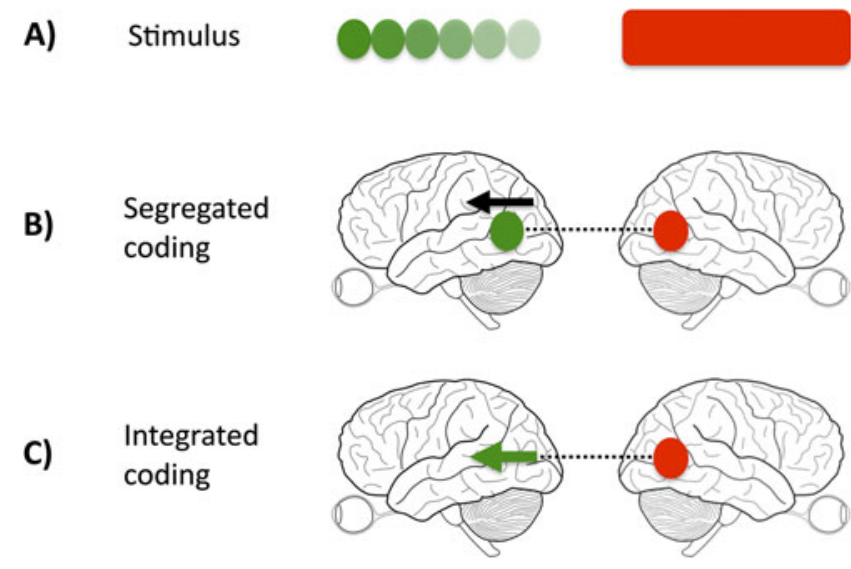

Fig. 1. Interaction of target and mask signals. (A) illustrates the physical stimulus, comprised of target and mask. (B) and (C) illustrate two possible ways, in which the visual cortex may represent the target and mask. In the case of a segregated representation (B), color and motion activate distinct and separate nodes (signified by the separate black arrow and green dot), whereas in the integrated case $(\mathrm{C})$, both direction of motion and color are represented within the same node (signified by the green arrow). A color-specific masking effect would support the existence of distinct processing nodes because the interference produced by the mask (dashed black line) acts only on the target color node.

opponent or nonopponent pairs. Fig. 2 shows the four target-mask color pairs.

In the first experiment, one short and one long SOA condition was tested (0-21 $\mathrm{ms}^{3}$ and $504 \mathrm{~ms}$, respectively). The long SOA is useful in ruling out confounding factors that could account for poor discrimination performance, such as general task difficulty or response confusion arising from the integration of target/mask information. Eighty trials per SOA were tested for each subject.

\section{Observers}

Ten subjects (average age 29 years; seven females) were tested on the initial version containing two different SOAs. All had normal or corrected to normal vision.

\section{Procedure}

Observers were instructed to report either the color or direction of motion (separate sessions) of the target and to ignore all features of the mask. The experiment used a two alternative forced-choice design and was performed in four sessions, run in a counterbalanced order. Each session was composed of blocks of 40 trials, with a break given after each. Observers completed a single practice block for each new task.

\section{Results}

Fig. 4A displays proportion-correct results for all conditions when a motion mask is used. At short SOAs, motion judgments are impaired (mean $=60 \%$ ), but this is not true of color judgments (mean $=95 \%$ ) where performance is at ceiling. A reversed pattern is

${ }^{3}$ Between subjects, performance at short SOAs was highly variable. To maintain a constant task difficulty, the short SOA was set on an individual subject basis, based on the results of three practice blocks (40 trials each) where SOA was set to 7, 14, and $21 \mathrm{~ms}$. One hundred and forty Hertz corresponds to a frame duration of $7.14 \mathrm{~ms}$. For reasons of clarity, we report SOAs rounded to the nearest millisecond. 
A

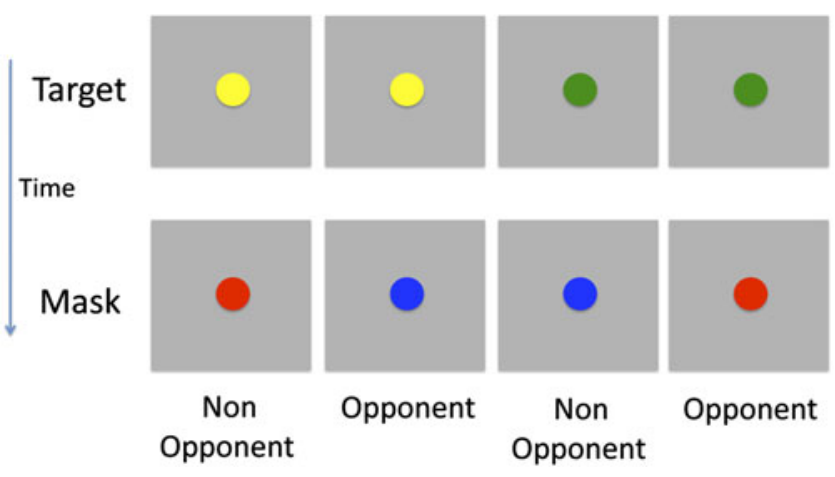

Fig. 2. Illustration of the different target and mask color pairs. The target was either yellow or green and the mask either red or blue. There the pairs consisted of either opponent or nonopponent colors. Note that the motion component of the stimulus is not shown.

shown in the complementary condition, employing a color mask (Fig. 4B).

Statistical comparison reveals a significant difference at short SOAs for both mask types (Motion mask: $t(11)=8.86, P<0.001$; Color mask: $t(11)=4.63, P<0.001)$, thus demonstrating a featureselective masking effect. Conversely, there is no significant difference in scores for the long SOA conditions [Motion mask: $t(11)=$ 1.48, $P=0.166$; Color mask: $t(11)=1.65, P=0.13$ ], for which masking was predicted to be minimal. Crucially, masking is not only significantly stronger within a visual dimension but is also weak or absent across dimensions. For judgments of color, the motion mask

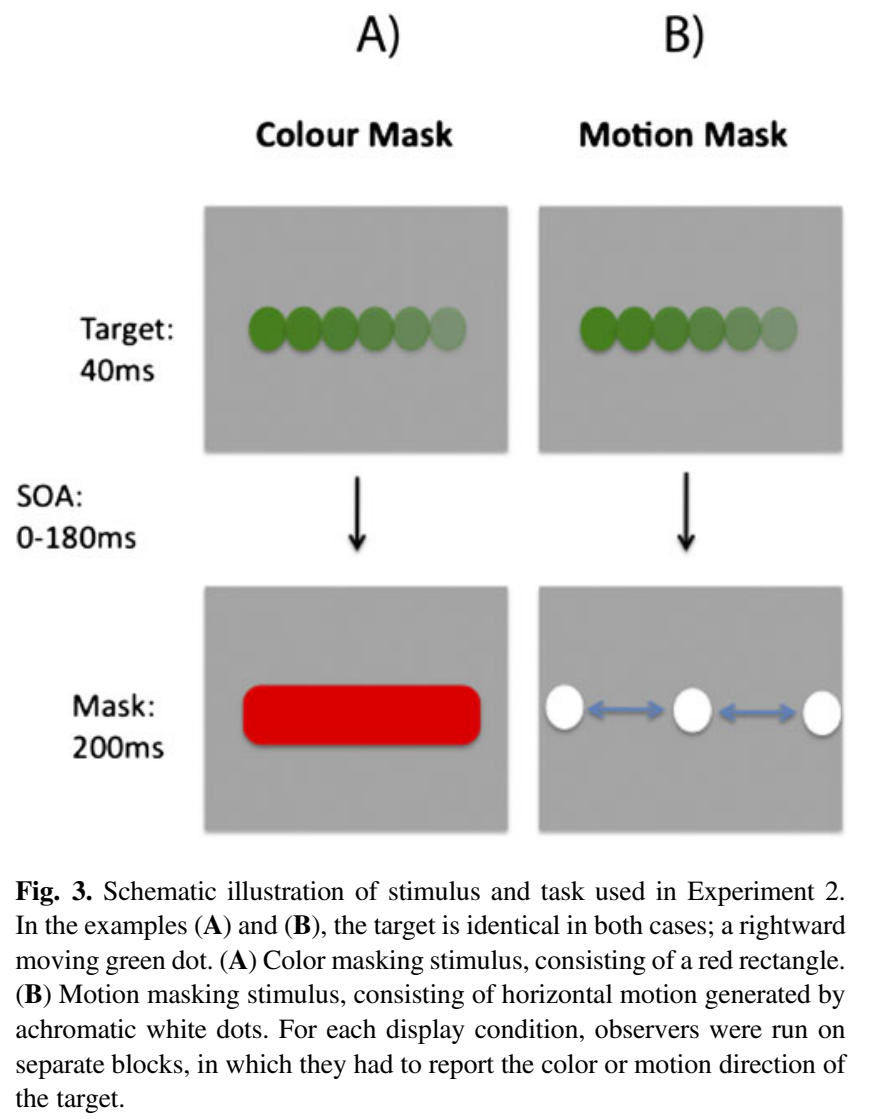

had little or no effect; performance remained at ceiling $(95 \%)$. Similarly, for judgments of motion, the color mask appears relatively ineffectual, although performance in this condition drops slightly $(<90 \%)$. Thus, for the display settings used in this experiment, it is possible to strongly mask one feature, while having no effect on the other.

In a separate analysis of the color masking data, we segregated trials into those containing opponent and nonopponent color pairs. The results failed to show a greater masking effect for opponent color pairs, $t(9)=1.7, P=0.13$.

\section{Experiment 2: Time course of the homogeneous masking effect}

Feature selectivity of visual masking, as demonstrated in Experiment 1 , lends clear support to the idea of segregated color and motion processing. Another method to investigate this separation is to examine differences in the masking time course. Previous studies, using a different paradigm, have argued for a faster color processing system than for motion, resulting in the generation of a color percept 70-80 ms before that of motion (Moutoussis \& Zeki, 1997a). Can this perceptual asynchrony be revealed using a masking paradigm? More specifically, is detectability of color greater than that of motion, at the same SOA, for masks of equal strength? In this experiment, we measure color and motion detectability for homogeneous target-mask pairs, using a range of different target and mask intervals (SOAs).

\section{Method}

\section{Observers}

Nine subjects (mean age 26 years; five females) were tested. All had normal or corrected to normal vision.

\section{Procedure}

Using the within dimension, stimuli described in Experiment 1, 10 different SOAs, from 7 to $142 \mathrm{~ms}$ (step size $\sim 15 \mathrm{~ms}$ ) ${ }^{4}$ were tested (48 trials per SOA). In order for a meaningful comparison of color and motion time courses to be made, it was important to first establish a benchmark, at which the color and motion masks were equally effective.

For each subject, using masked stimuli with a constant SOA of $21 \mathrm{~ms}$, mask strength yielding $60 \%$ correct was established through the use of an adaptive staircase procedure. Mask strength was varied by increasing or decreasing the luminance of the mask. This was done for both types of homogeneous target-mask pairs (colorcolor and motion-motion). Each subject's mask luminance values were then transferred to the main program measuring detectability at multiple SOAs.

\section{Results}

Fig. 5 shows detectability of the target feature (color or motion) as a function of SOA (target-mask interval) for an individual subject (Fig. 5A) and averaged across subjects (Fig. 5B). Performance for the second SOA value $(21 \mathrm{~ms})$ is approximately equal for color and motion conditions, indicating that mask strength has been successfully equalized (see Method for details). The idealized psychometric

\footnotetext{
${ }^{4}$ The SOA values are accurate to within \pm 3 ms due to MatLab/Cogent display limitations.
} 


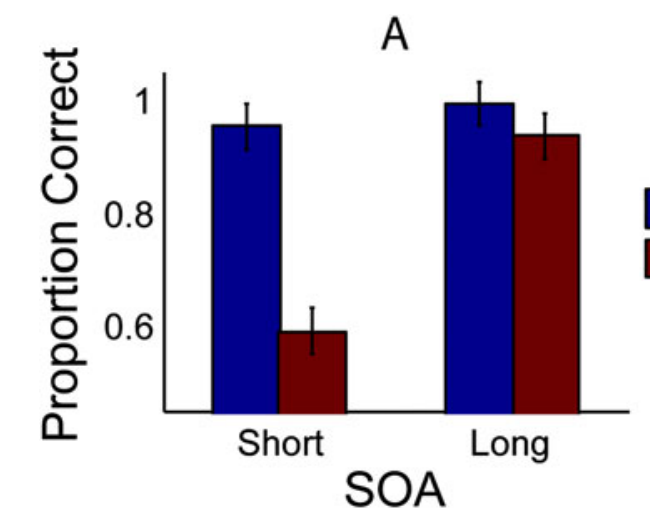

B

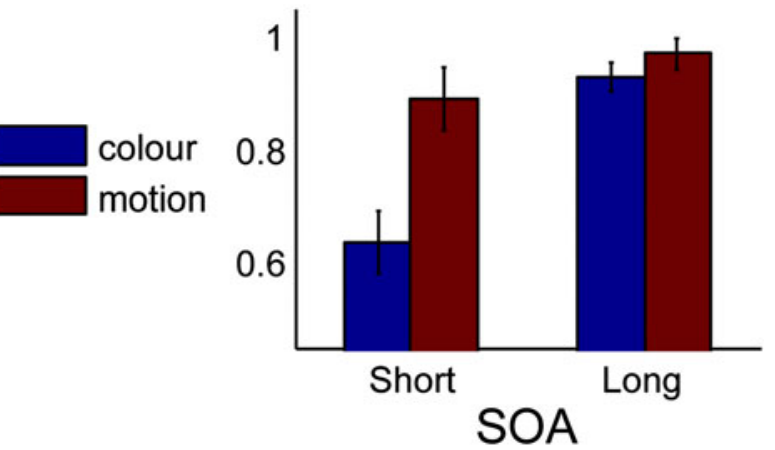

Fig. 4. Proportion correct for all observers $(n=12)$, for two SOA conditions (short and long), and judgements of either color (first column, blue) or motion (second column, red). Panel (A) displays the case where a motion mask is used. Panel (B) displays the case where a color mask is used. Error bars represent 1 standard error (SE) (within subjects).

function fitted to the data (Fig. 5A) demonstrates that color detectability increases more rapidly than motion detectability; color detectability plateaus at ceiling level by $150 \mathrm{~ms}$ SOA, while motion detectability continues to increase. Collapsed across the two longest SOA conditions of the group data (Fig. 5C), there is a significant difference between color and motion detectability, $t(8)=3.85, P<0.01$. This difference is not present at short SOAs, $t(8)=0.06, P>0.5$. These time course differences support a segregated processing scheme for color and motion, with color being processed more rapidly.
A

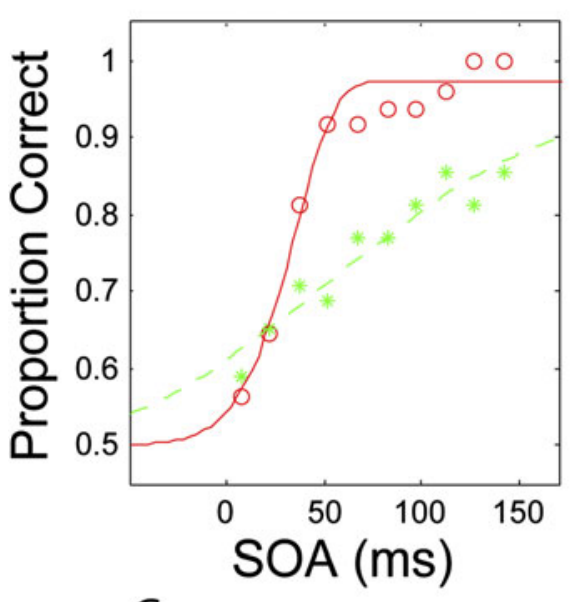

C
B

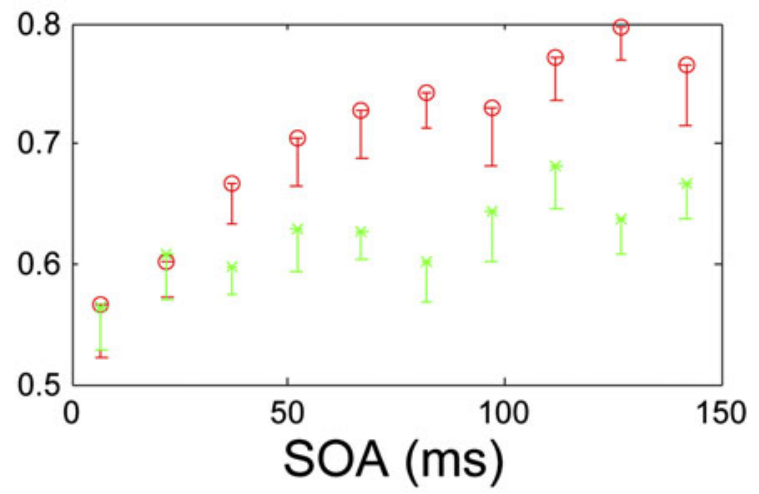

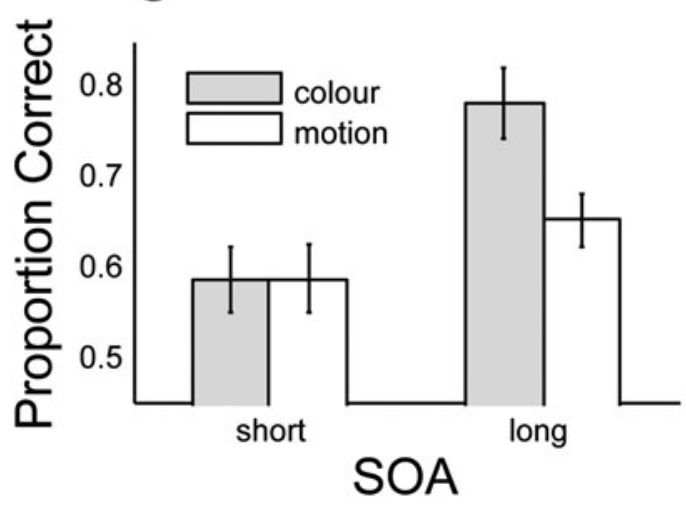

Fig. 5. Time course of heterogeneous masking for both color (red circles) and motion (green stars). (A) displays the data of a typical subject fitted with an idealized psychometric curve (Weibull function; Wichmann \& Hill, 2001). (B) displays mean data averaged across all subjects $(n=9)$. Error bars denote $1 \mathrm{SE}$. (C) shows detectability of the color or motion target at both short and long SOAs. The short condition was generated by collapsing across the two shortest SOA conditions, and the long condition was generated by collapsing across the two longest SOA conditions. 


\section{Experiment 3: Extending the time course: Forward and backward masking}

Experiment 1 demonstrated the existence of feature-selective masking using a backwards masking paradigm. In the Experiment 3, we wanted to learn if this pattern also applies to conditions when the mask is presented before the target (forward masking). Because binding requires more time when the signals to be bound are of a different type (e.g., color and motion; Bartels \& Zeki, 2006), we predicted that different target-mask types would require longer to interact, and that we would therefore see a strong cross-dimensional masking effect when the mask appears before, but not after, the target.

Method

\section{Observers}

Five observers (mean age 28 years; 1 female) were tested. All had normal or corrected to normal vision.

\section{Procedure}

In order to achieve greater flexibility in the temporal relationship between target and mask, and to test a finer scaled range of SOAs, the duration of the mask was decreased from 200 to $100 \mathrm{~ms}$. Observers were tested on two forward masking and two backward masking SOA conditions. In the forward masking conditions, the mask offset preceded the target by either 100 (Forward Long: FL) or $7 \mathrm{~ms}$ (Forward Short: FS). In the backward masking conditions, the mask onset succeeded the target by either 100 (Backwards Long: BL) or $7 \mathrm{~ms}$ (Backwards Short: BS). For each of these SOAs, observers were tested on all four color-motion target-mask combinations (see
Experiment 1 for details), using identical stimuli to Experiment 1. In total, each observer completed 80 trials per single condition.

\section{Results}

Fig. 6 displays overall proportion correct scores for all conditions, averaged across the five observers. The outer bars illustrate scores for the conditions, in which the mask and target had the greatest temporal separation. For these conditions, it was expected that masking would be minimal. The central two gray bars (conditions $F S$ and $B S$ ) represent the cases, in which the target is immediately preceded or succeeded by the mask.

\section{Heterogeneous masking}

When target and mask were of different features (Fig. 6A and $6 \mathrm{~B}$ ), performance for the longest SOAs (outer bars) approaches ceiling level (90\%) and masking is weak or absent. For the short SOAs there is a large masking effect when the mask immediately precedes that target $(F S)$; performance is significantly reduced in the forward $(F S)$ versus backward masking condition $(B S)$, for both the color mask / motion judgment $[t(4)=5.7, P<0.001]$ and motion mask / color judgment $[t(4)=2.5, P<0.05]$ conditions.

\section{Homogeneous masking}

Overall performance for within dimension masking (69\%) is less than that for across dimension masking (85\%), consistent with the results of Experiment 1. In common with the cross-dimension conditions, higher performance is seen for the longest SOAs (Fig. 6C and 6D, outer bars). In contrast to the cross-dimension

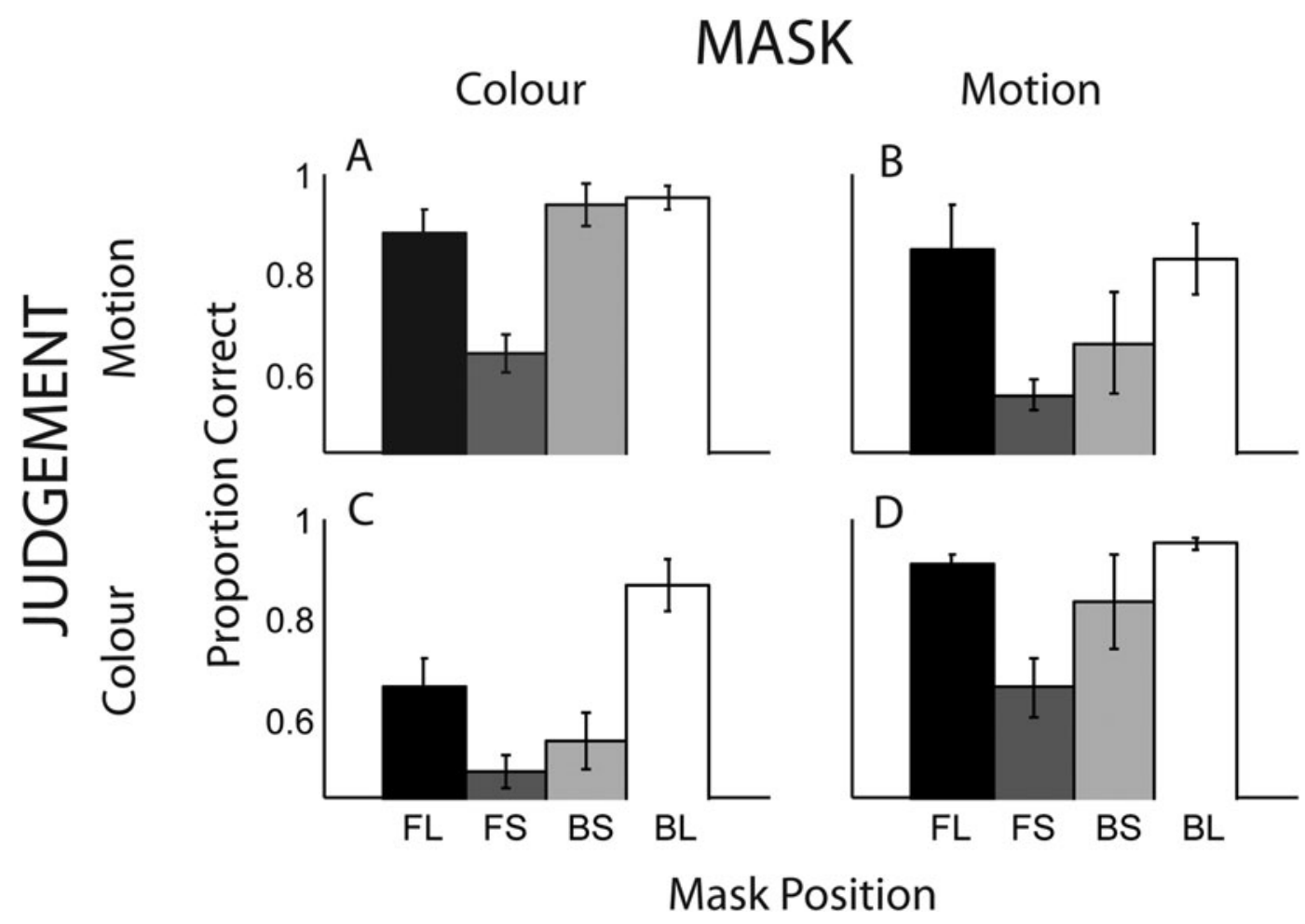

Fig. 6. Proportion correct for detection of either the color or direction of motion of a masked target. Each graph displays the four SOA conditions of FL, FS, BS, and BL (see main text for details). (A) Motion mask-color judgment, (B) color mask-motion judgment, (C) color mask-color judgment, and (D) motion mask-motion judgment. 
conditions, there is no significant difference between the short SOA conditions ( $F S$ and $B S$ ) for the color mask / color judgment condition, $t(4)=1.04, P=0.35$, and for the motion mask / motion judgment condition, $t(4)=0.97, P=0.39$.

A significant interaction between mask position (using the short SOA conditions of $F S$ and $B S$ ) and target-mask relationship (either across or within dimensions) provides strong evidence for dissociable masking time courses, $F(1,4)=17.0, P=0.015$. These results indicate that when the target and mask are of different features, presentation of the mask prior to the target is optimal for maximizing the strength of the mask. When the mask is presented subsequent to the target, the masking effect is weak. This finding is in agreement with the results of Experiments 1 and 2, which showed weak or absent heterogeneous backwards masking.

\section{Discussion}

Three variations on the visual masking paradigm have provided converging psychophysical support for the existence of functionally specialized color and motion systems in the human visual brain. It has been shown that 1) masking is feature selective, 2) color and motion recover at different rates from mask interference, and 3) the optimal temporal position of the mask is dependent on the feature relation of the target-mask pair. All three lines of evidence point toward a segregated coding scheme for color and motion in the human visual system.

Although feature-selective visual masking is a logical consequence of functionally specialized color and motion systems, this separation has not been extensively explored with psychophysical techniques before. This was the main focus of Experiment 1, in which we show that when a color mask is presented, color judgments are impaired, while motion judgments are spared. When a motion mask is presented, the reverse is true. The selective masking effect is apparent only when the mask is presented subsequently to the target (backwards masking), is significant only at short SOAs, and disappears by $500 \mathrm{~ms}$, consistent with previous masking results (Breitmeyer \& Ogmen, 2006) and ruling out the influence of other factors such as response confusion or memory limitations. A more in-depth examination of the time course of the masking effect was carried out in Experiments 2 and 3. The finding that detectability of the target color increases more rapidly than for motion (Experiment 2) is consistent with a shorter perceptual processing time for color compared to motion (Moutoussis \& Zeki, $1997 a$ ), and therefore, a shorter time window in which interference from the mask signal is effective. Unequal processing times for color and motion imply that their perceptual encoding is accomplished by different neurons in the visual system. Forward masking is not feature selective but takes place with any combination of target and mask (Experiment 3). This implies that heterogeneous masking can be effective, but the target and mask signals may require more time to interact. This account fits well with the known functional segregation of the visual system and is also supported by evidence that binding across feature dimensions requires more time (Bartels \& Zeki, 2006). Additionally, we found that masking strength does not depend on opponent/nonopponent target-mask color pairs.

The results of these experiments add to previous evidence demonstrating functional specialization in the vision system (Zeki, 1978; DeYoe \& van Essen, 1988; Livingstone \& Hubel, 1988). They do not rule out the existence of cells in the visual system that respond to multiple properties, as has been reported for color and form (Friedman et al., 2003) and for color and motion (Leventhal et al., 1995; Seymour et al., 2009) but only that such units, assuming them to exist, do not display their perceptual potency in these experiments. Therefore, although these results point to the importance of separate processing streams, they are not in themselves at odds with the existence of conjunction detectors for multiple visual properties, as previously reported for color and form (Lovegrove \& Over, 1973; Lovegrove \& Badcock, 1981; Clifford et al., 2003) and color and motion (Seymour et al., 2009). The observation that there was little or no carryover effect (color masking motion or vice versa) indicates that any conjunction-selective cells contribute only weakly to perception, if at all. This of course raises the question of what the role of such putative conjunction-selective cells may be.

It is worth noting that for heterogeneous masking (Experiment 3), the type of target-mask pair made no difference. Regardless of whether color-masked motion or motion-masked color, the largest effect was found when the mask preceded that target by $100 \mathrm{~ms}$. It is possible, therefore, that the processing latency differences for color and motion (Moutoussis \& Zeki, 1997a) relate only to the development of conscious percepts. Interference from the mask may take place before conscious percepts are generated.

\section{References}

Ansorge, U., Francis, G., Herzog, M. \& Ögmen, H. (2007). Visual masking and the dynamics of human perception, cognition, and consciousness. A century of progress, a contemporary synthesis, and future directions. Advances in Cognitive Psychology 3, 1-8.

Arnold, D.H., Clifford, C.W. \& Wenderoth, P. (2001). Asynchronous processing in vision: color leads motion. Current Biology 11, 596-600.

Barbur, J.L., Wolf, J. \& Lennie, P. (1998). Visual processing levels revealed by response latencies to changes in different visual attributes. Proceedings of the Royal Society of London. Series B, Biological Sciences 265, 2321-2325.

BARTELS, A. \& ZEKI, S. (2006). The temporal order of binding visual attributes. Vision Research 46, 2280-2286.

BRADDICK, O. (1973). The masking of apparent motion in random-dot patterns. Vision Research 13, 355-369.

Breitmeyer, B. \& Ogmen, H. (2006). Visual Masking: Time Slices through Conscious and Unconscious Vision (Oxford Psychology Series). Oxford: Oxford University Press.

Breitmeyer, B.G., Ro, T. \& Singhal, N.S. (2004). Unconscious color priming occurs at stimulus- not percept-dependent levels of processing. Psychological Science 15, 198-202.

Cavanagh, P., Tyler, C.W. \& Favreau, O.E. (1984). Perceived velocity of moving chromatic gratings. Journal of the Optical Society of America. A, Optics and Image Science 1, 893-899.

Clifford, C.W.G., Spehar, B., Solomon, S.G., Martin, P.R. \& Zaidi, Q. (2003). Interactions between color and luminance in the perception of orientation. Journal of Vision 3, 106-115.

DeYoe, E.A. \& Van Essen, D.C. (1988). Concurrent processing streams in monkey visual cortex. Trends in Neurosciences 11, 219-226.

DobKins, K.K. \& AlbRight, T. (1994). What happens if it changes colour when it moves?: The nature of chromatic input to macaque visual area MT. The Journal of Neuroscience 14, 4854-4870.

FERrera, V. \& Wilson, H. (1987). Direction specific masking and the analysis of motion in two dimensions. Vision Research 27, 1783-1796.

Friedman, H.S., Zhou, H. \& VON DER HeydT, R. (2003). The coding of uniform colour figures in monkey visual cortex. The Journal of Physiology 548, 593-613.

Gouras, P. \& Kruger, J. (1979). Responses of cells in foveal visual cortex of the monkey to pure colour contrast. Journal of Neurophysiology $\mathbf{4 2}$, $850-860$.

Hong, S.W. \& BLAKE, R. (2009). Interocular suppression differentially affects achromatic and chromatic mechanisms. Attention, Perception \& Psychophysics 71, 403-411.

Hong, S.W. \& SHEvelL, S.K. (2006). Resolution of binocular rivalry: Perceptual misbinding of color. Visual Neuroscience 23, 561-566.

KrumhansL, C.L. (1984). Independent processing of visual form and motion. Perception 13, 535-546.

Leventhal, A.G., Thompson, K.G., Liu, D., Zhou, Y. \& Ault, S.J. (1995). Concomitant sensitivity to orientation, direction, and color of 
cells in layers 2,3 , and 4 of monkey striate cortex. The Journal of Neuroscience 15, 1808-1818.

Livingstone, M.S. \& Hubel, D.H. (1987). Psychophysical evidence for separate channels for the perception of form, color, movement, and depth. The Journal of Neuroscience 7, 3416-3468.

Livingstone, M. \& Hubel, D. (1988). Segregation of form, color, movement, and depth: Anatomy, physiology, and perception. Science 240, 740-749.

LOVEGROVE, W. \& BADCOCK, D. (1981). The effect of spatial frequency on colour selectivity in the tilt illusion. Vision Research 21, 1235-1237.

Lovegrove, W. \& Over, R. (1973). Colour selectivity in orientation masking and aftereffect. Vision Research 13, 895-901.

Meadows, J.C. (1974). Disturbed perception of colours associated with localized cerebral lesions. Brain 97, 615-632.

Moutoussis, K. \& ZeKI, S. (1997a). A direct demonstration of perceptual asynchrony in vision. Proceedings of the Royal Society of London. Series B, Biological Sciences 264, 393-399.

Moutoussis, K. \& ZeKI, S. (1997b). Functional segregation and temporal hierarchy of the visual perceptive systems. Proceedings of the Royal Society of London. Series B, Biological Sciences 264, 1407-1414.

RAMACHANDRAN, V.S. \& GREgory, R.L. (1978). Does colour provide an input to human motion perception? Nature 275, 55-56.

Schmidt, T. (2002). The finger in flight: Real-time motor control by visually masked color stimuli. Psychological Science 13, 112-118.
Seymour, K., Clifford, C.W., Logothetis, N.K. \& Bartels, A. (2009). The coding of color, motion, and their conjunction in the human visual cortex. Current Biology 19, 177-183.

Theeuwes, J. (1992). Perceptual selectivity for color and form. Perception \& Psychophysics 51, 599-606.

WichmanN, F.A. \& HiLL, N.J. (2001). The psychometric function: I. Fitting, sampling, and goodness of fit. Perception \& Psychophysics 63, 1293-1313.

ZeKI, S. (1978). Functional specialisation in the visual cortex of the rhesus monkey. Nature 274, 423-428.

ZEKI, S. (1990). A century of cerebral achromatopsia. Brain 113, 1721-1777.

ZeKI, S. (1991). Cerebral akinetopsia (visual motion blindness). A review. Brain 114, 811-824.

ZeKI, S. \& Moutoussis, K. (1997). Temporal hierarchy of the visual perceptive systems in the Mondrian world. Proceedings of the Royal Society of London. Series B, Biological Sciences 264, 1415-1419.

ZeKI, S. \& ShIPP, S. (1988). The functional logic of cortical connections. Nature 335, 311-317.

Zeki, S., Watson, J.D., Lueck, C.J., Friston, K.J., Kennard, C. \& FrackowiaK, R.S. (1991). A direct demonstration of functional specialization in human visual cortex. The Journal of Neuroscience 11, 641-649.

Zihl, J., von Cramon, D., MaI, N. \& Schmid, C. (1991). Disturbance of movement vision after bilateral posterior brain damage. Further evidence and follow up observations. Brain 114(Pt 5), 2235-2252. 